\title{
Перераспределение оптической мощности в плечах волноводного Y-разветвителя при локальной внешней засветке подложки ниобата лития
}

\author{
(C) М.В. Парфренов ${ }^{1,2}$, А.В. Тронев ${ }^{1,3}$, И.В. Ильичев ${ }^{1}$, П.М. Агрузов ${ }^{1}$, А.В. Шамрай ${ }^{1}$ \\ ${ }^{1}$ Физико-технический институт им. А.Ф. Иофрфе РАН, Санкт-Петербург, Россия \\ ${ }^{2}$ Санкт-Петербургский политехнический университет Петра Великого, Санкт-Петербург, Россия \\ ${ }^{3}$ Санкт-Петербургский национальный исследовательский университет информационных технологий, механики и оптики \\ (Университет ИТМО), Санкт-Петербург, Россия \\ E-mail: mvparfenov@yandex.ru
}

Поступило в Редакцию 16 сентября 2019 г.

В окончательной редакции 16 сентября 2019г.

Принято к публикации 26 сентября 2019 г.

\begin{abstract}
Исследована оптическая перестройка коэффициента деления мощности в интегрально-оптическом $Y$ разветвителе, изготовленном на подложке фоторефрактивного кристалла ниобата лития $\left(\mathrm{LiNbO}_{3}\right)$. Определены области $Y$-разветвителя, наиболее чувствительные к локальной внешней засветке. Экспериментально продемонстрирована перестройка коэффициента деления на $2 \%$, что может быть эффективно использовано для повышения контраста модуляции в модуляторах Маха-Цендера.
\end{abstract}

Ключевые слова: интегральная оптика, фоторефрактивный эффект, оптический модулятор, коэффициент контраста, $Y$-разветвитель.

DOI: $10.21883 /$ PJTF.2020.01.48855.18040

Волноводные делители оптической мощности являются одними из базовых элементов интегральной оптики [1], а их характеристики во многом определяют параметры интегрально-оптических устройств, в состав которых они входят. Одно из наиболее широко используемых интегрально-оптических устройств - модулятор Маха-Цендера [2], где делители оптической мощности применяются для деления между плечами интерферометра Маха-Цендера и последующего сведения оптических волн вместе. В идеальном случае, если оптические волны, интерферирующие на выходе модулятора, имеют одинаковые амплитуды, контраст модуляции интенсивности стремится к бесконечности [3]. В реальных модуляторах такого типа значение коэффициента контраста лежит в пределах 20-30 dB и определяется дефектами, возникающими при изготовлении волноводных элементов интегрально-оптического интерферометра. В то же время для современных практических приложений, связанных с распределением квантовых ключей [4] и опросом распределенных систем прецизионных волоконнооптических датчиков [5], контраст модуляции должен быть на уровне $50 \mathrm{~dB}$ и выше.

Одним из предложенных авторами методов повышения характеристик интегрально-оптических схем, изготовленных на подложке ниобата лития $\left(\mathrm{LiNbO}_{3}\right)$, является внесение в их топологию компенсирующих дефектов [6]. Для этих задач можно использовать фоторефрактивный эффект, локальное возбуждение которого в подложке ниобата лития уменьшает показатель преломления на величину порядка $10^{-3}$ [7]. Работоспособность метода была показана на примере изменения коэффициента деления направленного $X$-ответвителя [6], работа которого основана на делении амплитуды за счет связи двух близкорасположенных оптических волноводов. В модуляторах Маха-Цендера обычно используют другой вид делителя оптической мощности $Y$-разветвитель. Принцип действия $Y$-разветвителя основан на эффекте деления волнового фронта, что делает его характеристики (в отличие от характеристик $X$-ответвителя) мало зависящими от длины волны света и изменения температуры. Отличаться будет и отклик на локальное изменение показателя преломления, который будет связан с возбуждением асимметричной моды.

Цель настоящей работы - провести теоретические и экспериментальные исследования фоторефрактивной перестройки коэффициента деления $Y$-разветвителя на подложке ниобата лития, определить наиболее чувствительные к локальному изменению показателя преломления места топологии и оценить возможное повышение контраста модуляции в интегрально-оптическом интерферометре Маха-Цендера при фоторефрактивной подстройке одного из $Y$-разветвителей.

Для исследований был выбран $Y$-разветвитель (рис. $1, a$ ) на основе оптических волноводов, изготовленных на монокристаллической подложке ниобата лития $(x$-срез) методом термической диффузии титана. Такие волноводы стабильны и имеют низкие оптические потери [8]. В общем случае они поддерживают распространение двух ортогонально поляризованных групп мод: TE- и ТМ-типа. Для оптимальной работы модулятора подбирают условия, при которых волноводы работают в одномодовом режиме, а при помощи интегрального 


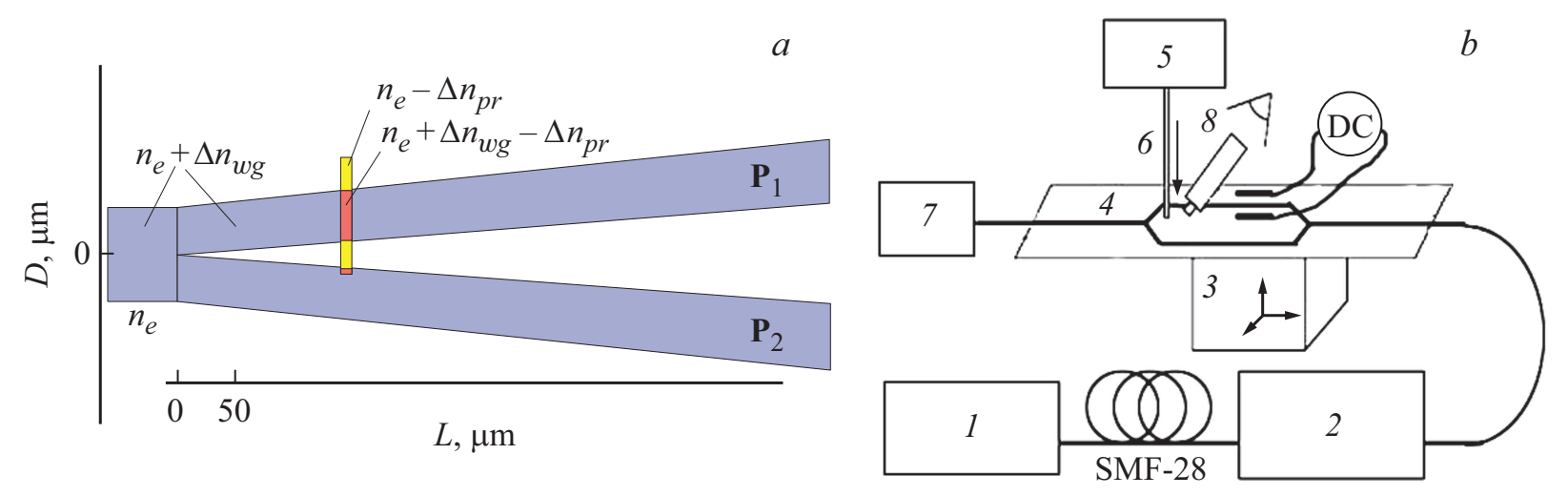

Рис. 1. $a-$ схема $Y$-разветвителя, использованного при моделировании; $b-$ экспериментальная установка: $1-$ DFB-лазер $(\lambda=1550 \mathrm{~nm}), 2$ - контроллер поляризации, 3 - трехосевая координатная подвижка, 4 - интегрально-оптический модулятор, $5-\mathrm{He}-\mathrm{Ne}$-лазер $(\lambda=633 \mathrm{~nm}), 6$ - одномодовое оптическое волокно, $7-$ фотоприемник, $8-$ микроскоп.
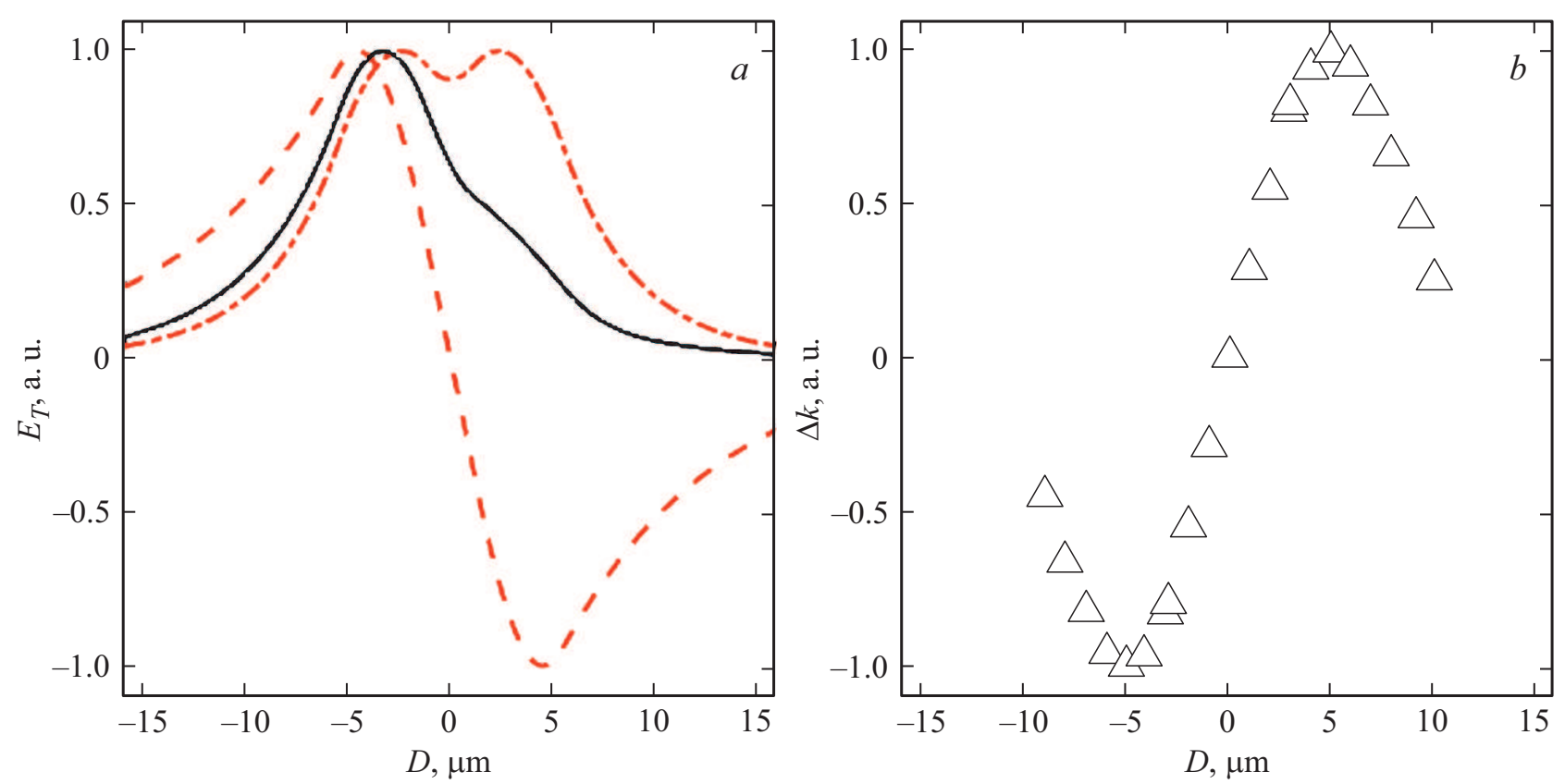

Рис. 2. $a$ - распределение тангенциальной напряженности поля мод по поперечной координате (штриховая и штрихпунктирная линии соответствуют модам до засветки, сплошная линия - моде после засветки); $b$ - зависимость рассчитанного изменения коэффициента деления $Y$-разветвителя от расположения области фоторефрактивной засветки по поперечной координате. Обе части рисунка соответствуют продольной координате $L=650 \mathrm{~nm}$.

поляризатора оставляют только ТЕ-моду [9], чувствительную к распределению необыкновенного показателя преломления.

Методом ВРМ (beam propagation method) было проведено численное моделирование и получены теоретические зависимости коэффициента деления мощности от расположения области локального уменьшения показателя преломления. Для упрощения моделирование проводилось для оптических волноводов со ступенчатым профилем показателя преломления и приращением относительно подложки $\Delta n_{w g}=2 \cdot 10^{-3}$. Область локальной внешней засветки представлялась в виде квадрат- ного пятна размером $10 \times 10 \mu \mathrm{m}$, вызывающего за счет фоторефрактивного эффекта равномерное уменьшение показателя преломления на $\Delta n_{p r}=1 \cdot 10^{-3}$ на всю толщину подложки. Положение пятна относительно топологии $Y$-разветвителя менялось по обеим координатам в плоскости подложки.

Основной параметр $Y$-разветвителя - коэффициент деления - высчитывался как

$$
k=\frac{P_{2}}{P_{1}+P_{2}}
$$

где $P_{1}$ и $P_{2}-$ значения мощности двух разнесенных несвязанных волноводов на выходе $Y$-разветвителя. 

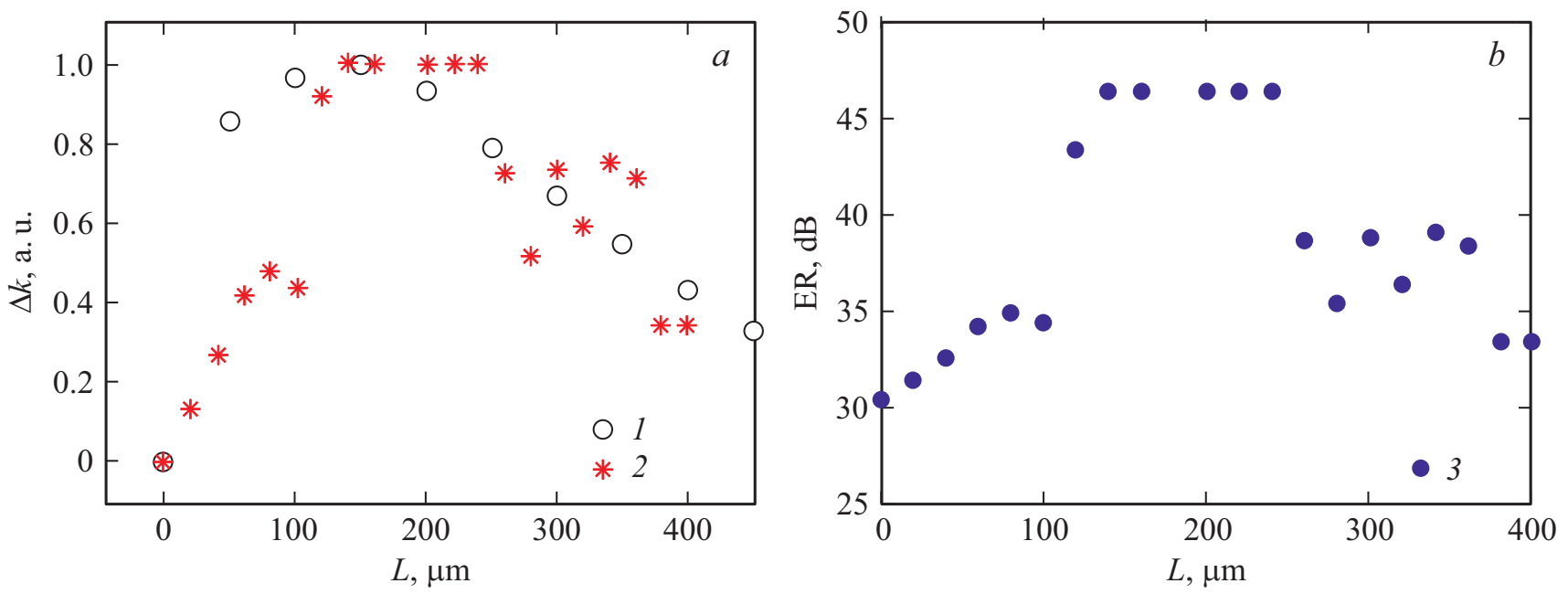

Рис. 3. Зависимость изменения коэффициента деления $Y$-разветвителя $(a)$ и коэффициента контраста $(b)$ от расположения области фоторефрактивной засветки по продольной координате. 1 - результаты моделирования, 2 и 3 - результаты эксперимента.

Результаты расчета показывают, что локальное уменьшение показателя преломления в одном из плеч $Y$-разветвителя приводит к уменьшению мощности в этом плече на выходе (рис. 2, $b$ и $3, a$ ). Оптимальное положение центра пятна локального изменения показателя преломления относительно топологии $Y$-разветвителя соответствует максимальной деформации профиля собственных мод (симметричной и асимметричной) в области ветвления. В продольном направлении относительно направления распространения света это точка максимального взаимодействия между плечами волноводов. В поперечном направлении она соответствует максимумам полей волноводных мод (рис. 2).

Экспериментальная перестройка коэффициента деления $Y$-разветвителя была реализована на установке, представленной на рис. $1, b$. Локальное облучение заданной области $Y$-разветвителя обеспечивалось при помощи оптического зонда, выполненного на основе телекоммуникационного оптического волокна типа SMF-28, обеспечивавшего облучение поверхности кристалла светом от $\mathrm{He}-\mathrm{Ne}$-лазера $(\lambda=633 \mathrm{~nm}$, мощность $P=2 \mathrm{~mW}$, площадь пятна $\left.\sim 10 \mu \mathrm{m}^{2}\right)$. Время экспозиции $t_{\text {exp }} \approx 300 \mathrm{~s}$ соответствовало достижению стационарного значения фоторефрактивного изменения показателя преломления. Позиционирование зонда осуществлялось при помощи трехкоординатной микроподвижки.

Образец представлял собой чип интегрально-оптического модулятора Маха-Цендера с интерферометром, образованным двумя $Y$-разветвителями. Осуществлялась фоторефрактивная подстройка разветвителя на входе модулятора. После локальной засветки проводились измерения коэффициента контраста модулятора. После проведения измерений образец возвращался к исходному состоянию при помощи мощного однородного ультрафиолетового излучения. Полученные экспериментальные результаты были пересчитаны в изменение коэффициента деления $Y$-разветвителя по формулам

$$
\begin{gathered}
k=\frac{1-\sqrt{1-\left(\frac{A-1}{A+1}\right)^{2}}}{2}, \\
A=10^{\mathrm{ER} / 10},
\end{gathered}
$$

где $\mathrm{ER}$ - значения коэффициента контраста модуляции (в dB). При этом для простоты предполагалось, что неидеальность интерферометра определялась исключительно неточностью деления мощности во входном $Y$-разветвителе.

Полученные экспериментальные результаты представлены вместе с результатом моделирования на рис. 3 . Несмотря на ряд сделанных допущений, наблюдается хорошее согласие результатов теоретического анализа с экспериментальными данными. Это показывает, что предложенная упрощенная модель адекватно описывает эффект фоторефрактивного управления $Y$-разветвителем. При этом область максимальной чувствительности $Y$-разветвителя приходится именно на область вблизи точки ветвления входного волновода на два, что соответствует качественным предположениям, сделанным ранее.

При выбранных параметрах перестройка коэффициента деления возможна в пределах до 1-2\%. Этого оказалось достаточно для повышения коэффициента контраста интегрально-оптического модулятора на его основе на существенные значения (10-17 dB при изначальном коэффициенте контраста $30 \mathrm{~dB}$ ).

Возможность производить перестройку коэффициента деления для уже изготовленных модуляторов с достижением значительного увеличения коэффициента контраста модуляции, отсутствие потребности в сложном оборудовании для выполнения процедуры и возможность стирать эффект в случае неудовлетворительного результата $[6,10]$ являются явными преимуществами 
метода и могут широко применяться для оптимизации характеристик модуляторов на основе ниобата лития.

\section{Финансирование работы}

Работа выполнена при поддержке Российского научного фонда (проект № 19-19-00511).

\section{Конфликт интересов}

Авторы заявляют, что у них нет конфликта интересов.

\section{Список литературы}

[1] Alferness R. // IEEE J. Quant. Electron. 1981. V. 17. N 6. P. 946-959.

[2] Петров В.М., Шамрай А.В. Интерференция и дифракция для информационной фотоники. СПб.: Лань, 2019. 460 с.

[3] Chen A., Murphy E.J. Broadband optical modulators: science, technology, and applications. Boca Raton: CRC Press, 2012. $532 \mathrm{p}$.

[4] Wang X., Liu J., Li X., Li Y. // IEEE J. Quant. Electron. 2015. V. 51. N 6. P. 5200206 (1-6).

[5] Bai Q., Wang Q., Wang D., Wang Y., Gao Y., Zhang H., Zhang M., Jin B. // Sensors (Basel). 2019. V. 19. N 8. P. 1862.

[6] Парфенов М.В., Тронев А.В., Ильичев И.В., Агрузов П.М., Шамрай А.В. // Письма в ЖТФ. 2019. Т. 45. В. 5. С. 3-5.

[7] Chen F.S. // J. Appl. Phys. 1969. V. 40. N 8. P. 3389-3396.

[8] Bazzan M., Sada C. // Appl. Phys. Rev. 2015. V. 2. N 4. P. 040603.

[9] Ильичев И.В., Тогузов Н.В., Шамрай А.В. // Письма в ЖТФ. 2009. Т. 35. В. 17. С. 97-103.

[10] Grachev A.I., Chamrai A.V., Petrov M.P. // OSA TOPS. 2011. V. 62. P. 203-211. 